\title{
BMJ Open Development of a meta-algorithm for guiding primary care encounters for patients with multimorbidity using evidence-based and case-based guideline development methodology
}

Cathleen Muche-Borowski, Dagmar Lühmann, Ingmar Schäfer, Rebekka Mundt, Hans-Otto Wagner, Martin Scherer, the Guideline Group of the German College of General Practice and Family Medicine (DEGAM)

To cite: Muche-Borowski C, Lühmann D, Schäfer I, et al. Development of a metaalgorithm for guiding primary care encounters for patients with multimorbidity using evidence-based and casebased guideline development methodology. BMJ Open 2017;7:e015478. doi:10.1136/ bmjopen-2016-015478

- Prepublication history and additional material is available. To view please visit the journal online (http://dx.doi.org/10. 1136/10.1136/bmjopen-2016015478)

CM-B and DL contributed equally.

Received 9 December 2016 Revised 21 March 2017 Accepted 21 March 2017

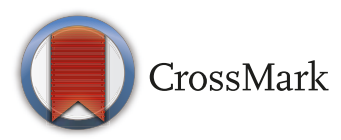

Institute for Primary Care and Family Medicine, University Medical Center HamburgEppendorf (UKE), Hamburg, Germany

Correspondence to Dr Cathleen Muche-Borowski; c.borowski@uke.de

\section{ABSTRACT}

Objective The study aimed to develop a comprehensive algorithm (meta-algorithm) for primary care encounters of patients with multimorbidity. We used a novel, case-based and evidence-based procedure to overcome methodological difficulties in guideline development for patients with complex care needs.

Study design Systematic guideline development methodology including systematic evidence retrieval (guideline synopses), expert opinions and informal and formal consensus procedures.

Setting Primary care.

Intervention The meta-algorithm was developed in six steps:1. Designing 10 case vignettes of patients with multimorbidity (common, epidemiologically confirmed disease patterns and/or particularly challenging health care needs) in a multidisciplinary workshop.2. Based on the main diagnoses, a systematic guideline synopsis of evidence-based and consensus-based clinical practice guidelines was prepared. The recommendations were prioritised according to the clinical and psychosocial characteristics of the case vignettes.3. Case vignettes along with the respective guideline recommendations were validated and specifically commented on by an external panel of practicing general practitioners (GPs).4. Guideline recommendations and experts' opinions were summarised as case specific management recommendations ( $\mathrm{N}$-ofone guidelines).5. Healthcare preferences of patients with multimorbidity were elicited from a systematic literature review and supplemented with information from qualitative interviews.6. All N-of-one guidelines were analysed using pattern recognition to identify common decision nodes and care elements. These elements were put together to form a generic meta-algorithm.

Results The resulting meta-algorithm reflects the logic of a GP's encounter of a patient with multimorbidity regarding decision-making situations, communication needs and priorities. It can be filled with the complex problems of individual patients and hereby offer guidance to the practitioner. Contrary to simple, symptom-oriented algorithms, the meta-algorithm illustrates a superordinate process that permanently keeps the entire patient in view.

\section{Strengths and limitations of the study}

- This is the first study to attempt a case-based 'bottom-up' approach to developing a guideline for patients with multimorbidity and complex care needs in primary care.

- A methodological approach consisting of quantitative and qualitative methods was used to combine research evidence, experts' opinions and patients' preferences.

- The meta-algorithm in its final form was formally consented by the multidisciplinary guideline group that is led by the German College of General Practice and Family Medicine.

- For reasons of convenience, the number of underlying case vignettes was limited to 10 , hereby narrowing the covered spectrum of multimorbidity.

- It cannot be excluded that our sample of general practitioners is a selection of excellence, and they might not be representative for the whole sample of all practicing primary care physicians caring for patients with multimorbidity.

Conclusion The meta-algorithm represents the back bone of the multimorbidity guideline of the German College of General Practitioners and Family Physicians. This article presents solely the development phase; the meta-algorithm needs to be piloted before it can be implemented.

\section{INTRODUCTION}

Background

Multimorbidity, defined as the presence of several chronic conditions in one person, is a very common phenomenon in the elderly. It is still difficult to quantify unequivocally how many people suffer from multimorbidity as there is no general consensus on the definition or measurement of multimorbidity. ${ }^{1}$ Which conditions contribute to multimorbidity and 
how many of them need to be present to constitute multimorbidity are particularly the controversially debated questions. The lack of a definition explains the large differences in reported prevalence figures that depend on the disease spectrum included, the setting and the data sources used and the time period assessed. ${ }^{2-5}$

The consequences of multimorbidity for the patients include functional disabilities, a lower quality of life, higher mortality, higher usage of the healthcare system and thus higher costs. ${ }^{16-8}$ The complex care needs of patients with multimorbidity present a particular challenge for the patients themselves and for their care providers. The best explored and most widely discussed care problem is the polypharmacy associated with multimorbidity. This phenomenon is characterised by incalculable interactions of medications and illnesses, adverse effects or contradictory therapeutic strategies. ${ }^{9} 10$ Furthermore, assistive non-pharmaceutical therapies, educational interventions, self-care measures and frequent follow-ups recommended by different individual disease guidelines contribute to the treatment burden of patients with multimorbidity. Patients with 12 different daily medications and 24 daily treatment routines-as demonstrated in the frequently cited case by Boyd et al ${ }^{11}$-are often encountered in primary care. ${ }^{11}$

Given the complexity of health problems in patients with multimorbidity, there is wide consensus that the concept of 'patient-centred care' should guide any approach to care. ${ }^{12}$ The central aspects of the concept include the pursuit of a biopsychosocial disease concept, the pivotal role of patients' central values and priorities, a doctor-patient relationship, shared decision making and a coordinated approach to interdisciplinary care. ${ }^{13} 14$

Muth et al in 2014 formulated a set of principles ('Ariadne principles') specifically for the general practice setting with the intention to guide primary care consultations of patients with multimorbidity. ${ }^{15}$ The principles follow the concept of patient-centred care and address the classic responsibilities of primary care: treating current problems, treating chronic problems, clarifying and coordinating patients' and doctors' expectations concerning treatment planning and opportunistic healthcare promotion. ${ }^{16}$

\section{Multimorbidity in clinical practice guidelines (CPG)}

So far, there is only one published CPG explicitly focusing on the care of patients with multimorbidity. ${ }^{17}$ The guideline published by the British National Institute of Excellence (NICE) takes a wide scope by addressing all healthcare professionals as well as patients, their families and caregivers. The German primary care guideline 'Multimedikation' (Polypharmacy) addresses medication for patients with multimorbidity but cannot depict the entire primary care process for this particular group of patients. ${ }^{18}$ CPG for single diseases rarely address multimorbidity. ${ }^{15171920}$ Applying the various recommendations of all applicable single disease guidelines is barely feasible and associated with a high risk for the patients due to interactions and incompatible treatments. ${ }^{11}$

Aside from the clinical complexity, guideline development for patients with multimorbidity bears methodological challenges. Five methodological steps are essential for the process of developing evidence-based and consensus-based CPG:

1. Assembly of a representative, interdisciplinary and multiprofessional guideline development group including experts, users and patients.

2. Identification of the clinically relevant key questions, which lead to the guidelines' main recommendations.

3. Systematic search for the best available empirical evidence to support the recommendations.

4. Appraisal of the available evidence from a clinical point of view, with a focus on relevant effects in daily practice and feasibility.

5. Structured, reproducible and independently moderated consensus rounds to finalise recommendations. ${ }^{2122}$

This classic, data-based and expert-based 'Top-Down' procedure has not proven particularly helpful in developing a guideline for the treatment of patients with multimorbidity due to the above-mentioned complexity. Using chronic heart failure and 18 common comorbidities as an example, Muth et al clearly outlined the various interactions between an index illness and a patient's comorbidities, diseasedrug interactions and drug-drug interactions (247 interactions, averagely 14 per comorbidity). ${ }^{23}$ All of these would need to be considered during the systematic evidence review in the Top-Down guideline development procedure. The implementation of such a procedure for multimorbidity does not seem feasible, especially without focusing on a particular index illness.

\section{OBJECTIVE}

Against this background, we elaborated and tested a 'bottom-up' procedure to develop a comprehensive algorithm to guide a primary care encounter of a patient with multimorbidity. The algorithm aims to give primary care encounters of patients with multimorbidity a structure. It is intended to support the setting of priorities in patients with complex care needs. The algorithm is intended to be the centrepiece of an evidence-based and consensus-based CPG 'Multimorbidity' (work in progress), created under the supervision of the German College of General Practitioners and Family Physicians (DEGAM) (http:// www.awmf.org/leitlinien/detail/anmeldung/1/1l/ 053-047.html). The multidisciplinary guideline panel was comprised of GPs, geriatricians, gerontologists, psychologists and health scientists with expertise in evidence-based medicine and guideline development. 


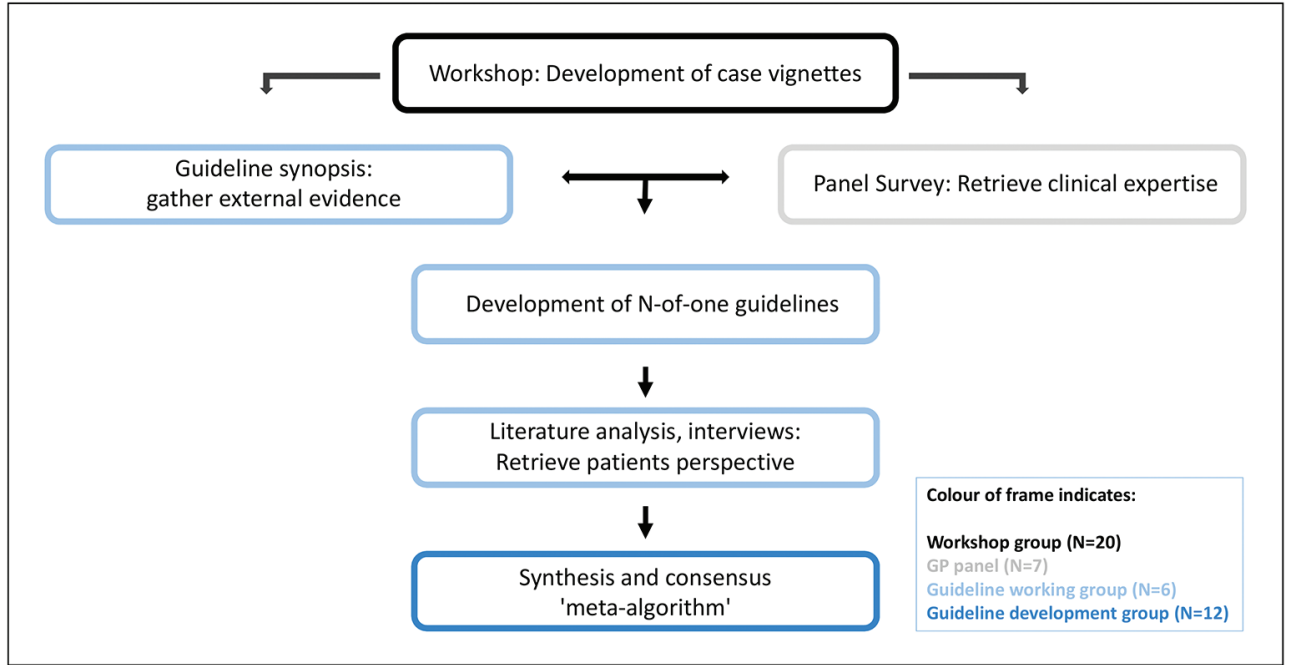

Figure 1 Methodological steps to develop a 'Meta-Algorithm' for the management of patients with multimorbidity.

\section{METHODS}

\section{General approach}

The 'bottom-up' procedure for guideline development is based on case vignettes of patients with multimorbidity. Individual management recommendations were generated for each case vignette, using standard guideline development methodology (problem identification/ formulation of key questions, evidence search and analysis, contextualisation and consensus finding). As in the 'N-of-one trials' (scientific studies with only one participant), ${ }^{24}$ the resulting recommendations were considered 'N-of-one guidelines'. Using a qualitative synthesis of the $\mathrm{N}$-of-one guidelines, a generic meta-algorithm was created that reflects management considerations for patients with multimorbidity in primary care. Figure 1 outlines the methodological steps and groups participating in the development process. The process was coordinated and largely realised by the guideline working group that consisted of three general practitioners and three experts in guideline development methodology.

In detail, six methodical steps were followed to develop the meta-algorithm for the management of patients with multimorbidity in primary care.

\section{Development of case vignettes}

Ten case vignettes of prototypic patients with multimorbidity consulting their GP were constructed in a single-day workshop with 20 interdisciplinary (GPs, methodologists, including the guideline working group) participants (workshop group). We chose 10 vignettes in order to be able to depict the most frequently seen multimorbidity patterns and disease combinations and be able to reflect particularly problematic combinations as seen by the GPs. Disease combinations for two-thirds of the vignettes were taken from epidemiological research: typical multimorbidity patterns (cardiovascular/metabolic, anxiety/depression/somatic disorders/pain-oriented morbidity and neuropsychiatric illnesses) reported in the Multicare Study ${ }^{25}$ and frequently encountered disease combinations published by van den Bussche et al. ${ }^{26}$
Disease combinations for one-third of the vignettes were derived from particularly difficult cases of multimorbidity presented by the participating general practitioners. Information on the clinical and psychosocial context of each case was collected based on the experience of the task force participants.

Each case vignette contained information on:

- demographics (age, gender and cccupation),

- the patient's medical history,

- reason for consultation, main complaints/health problem and symptoms,

- diagnoses thatconstitute the patient's 'Multimorbidity',

- psychosocial context (eg, marital status, housing situation, and so on),

- results of current examinations (clinical examinations or blood work),

- medications (name, dosage, application form and prescription data),

- psychosocial status.

\section{Evidence search and analysis}

The evidence base used in creating the management recommendations for each case vignette was derived from published evidence-based and consented CPG. A guideline synopsis was prepared for each case vignette including recommendations from guidelines that address the diagnoses that constitute multimorbidity in the respective vignette. In May 2013, the national guideline databases of the Association of Scientific Medical Societies (AWMF) and of the Agency for Quality in Medicine (AQuMed) were searched using the main diagnoses as search terms to create this base of evidence. Guidelines were included if they were up-to-date and contained a systematic work-up of the research evidence (see online supplementary file 1). In a first step, all guideline recommendations that applied to the main diagnoses of the case vignettes and that addressed aspects of long-term care of chronic illnesses were extracted by the scientific staff of the guideline working group. Recommendations 
referring to diagnostic procedures or emergency treatments were not considered. In a second step, the relevant guideline recommendations were identified and prioritised for each case vignette in a modified Delphi procedure by the clinical members of the guideline working group. Recommendations that seemed applicable-taking into consideration demographics, main ailments, psychosocial context, current medication and any additional information to the patient's current life situation-were added to the case vignettes.

\section{Adding clinical expertise}

All case vignettes and guideline recommendations were clinically validated by practicing GPs (GP panel). The GPs were recruited from the server list of an email-based professional discussion forum led and used by about 700 practicing primary care physicians from all over Germany. The members of the server's list were asked if they were interested in participating in a research project pertaining to the healthcare of patients with multimorbidity. All interested physicians received 10 electronic documents containing the case vignettes, a summary of the guideline recommendations and a questionnaire. The questionnaire consisted of three open-ended questions: (1) the necessity to avert life-threatening conditions, (2) the definition of management goals and (3) relevant lifestyle and psychosocial factors to be taken into consideration. Additionally, GPs were asked whether they referred to the guideline recommendations contained in the case vignettes when answering the questions. Participants were offered a compensation of $100 €$.

\section{Developing $\mathrm{N}$-of-one guidelines}

In a next step, the primary care process for each case vignette was displayed as an algorithmic graph (N-of-one guideline) based on guideline recommendations and clinical judgement. Each N-of-one guideline starts off with the reason for the patient's current consultation. The key questions guiding the care process are generated from the case vignettes with their heterogeneous multimorbidity constellations and psychosocial contexts. The recommendations referring to the key questions are derived from the external evidence (guideline recommendations) and the GP panel comments. All N-of-one guidelines were finalised in an informal consensus procedure within the guideline working group.

\section{Bringing in the patient's perspective}

Taking the values and preferences of the affected patient group into consideration is an essential step in guideline development. ${ }^{21}$ Two approaches were taken in order to clarify the preferences and values of patients with multimorbidity regarding their healthcare: (1) a systematic review of qualitative and quantitative studies and (2) qualitative interviews with 15 patients with multimorbidity sampled from the Multicare cohort study. ${ }^{27}$ This process was part of a dissertation project (RM) that will be published separately. The main methodological aspects and results are documented in the online supplementary file 2.
For the systematic literature review, Medline and Embase were searched via OVID, starting from inception until March 2015. In order to be included into the review, publications had to contain qualitative or quantitative information elicited from patients with multimorbidity regarding their preferences and values in healthcare. The methodological quality of the qualitative and quantitative studies was checked using the Giacomini and Cook ${ }^{28}$ as criteria for qualitative research and a modified Checklist of the Scottish Intercollegiate Guideline Network ${ }^{29}$ for quantitative research. Information from the quantitative studies was extracted into Excel sheets and summarised qualitatively, hereby identifying relevant categories and subcategories. Information from the qualitative materials was sorted into the same categories and integrated into the summaries. If necessary, new categories were defined.

For the qualitative interviews, the patients were matched to the case vignettes as closely as possible (for age, gender and comorbidities). The interview questions were sorted according to the rundown of a practice consultation, and the content analysis was based on the categories identified by the literature analysis (doctor-patient relationship, communication, therapeutic goals, subjective needs and framework conditions).

\section{Synthesis of the meta-algorithm}

The final product, the generic meta-algorithm to guide primary care consultations of patients with multimorbidity, was derived from the $10 \mathrm{~N}$-of-one guidelines in an informal, qualitative, synthesis procedure. All N-of-one guidelines were reviewed with the goal of identifying common key questions, decision-making processes, necessary information resources, healthcare consequences, patient preferences and context considerations ("pattern recognition'). Common elements and interconnections were reformulated, generalised (not related to a particular patient) and combined to create an algorithm that structures a primary care consultation of a patient with multimorbidity. The final version of the meta-algorithm was consented in a nominal group process by the multidisciplinary guideline development group that is led by the DEGAM.

\section{RESULTS}

\section{Case vignettes of patients with multimorbidity}

Ten case vignettes were established within the 1-day workshop by the multidisciplinary working group. Seven vignettes were based on epidemiologically confirmed disease patterns, ${ }^{25} 26$ and three vignettes were based on real patients with highly complex multimorbidity reported by the participating GPs (see table 1). In these cases, multimorbidity is constituted by clearly defined diseases and by symptoms such as fatigue or gait disturbance. The patients' ages and psychosocial backgrounds in all vignettes were contributed by the GPs who reflected on situations likely to complicate medical care, self-management and/or communication. 
Table 1 Overview of case vignettes

\begin{tabular}{|c|c|c|}
\hline Demography & Psychosocial context & Diagnoses \\
\hline \multicolumn{3}{|c|}{ Epidemiologically confirmed disease patterns ${ }^{25}$} \\
\hline $\begin{array}{l}91 \text { years, male } \\
\text { (figure 1) }\end{array}$ & $\begin{array}{l}\text { His wife suffered a stroke; the couple } \\
\text { lives secluded }\end{array}$ & $\begin{array}{l}\text { Depression, dementia, coronary heart disease (+ } \\
\text { urinary incontinence and hearing loss) }\end{array}$ \\
\hline $\begin{array}{l}66 \text { years, female } \\
\text { (see online supplementary file } 3 \text { ) }\end{array}$ & Retired, no further information & Chronic back pain, osteoporosis, headaches \\
\hline $\begin{array}{l}82 \text { years, female } \\
\text { (see online supplementary file 4) }\end{array}$ & Immigrated, speaks no German & $\begin{array}{l}\text { Chronic heart failure, chronic kidney failure, low } \\
\text { blood pressure }\end{array}$ \\
\hline $\begin{array}{l}55 \text { years, female } \\
\text { (see online supplementary file 5) }\end{array}$ & $\begin{array}{l}\text { Immigrated, familial problems, } \\
\text { does not speak German well }\end{array}$ & $\begin{array}{l}\text { High blood pressure, type } 2 \text { diabetes mellitus, } \\
\text { metabolic syndrome }\end{array}$ \\
\hline \multicolumn{3}{|c|}{ Most frequently encountered disease combinations ${ }^{26}$} \\
\hline $\begin{array}{l}82 \text { years, male } \\
\text { (see online supplementary file 6) }\end{array}$ & $\begin{array}{l}\text { Widowed, lives alone, daughter visits } \\
\text { every } 4 \text { weeks }\end{array}$ & High blood pressure, atrial fibrillation, depression \\
\hline \multicolumn{3}{|c|}{ Highly complex real cases, reported by participating GPs } \\
\hline $\begin{array}{l}76 \text { years, male } \\
\text { (see online supplementary file 9) }\end{array}$ & $\begin{array}{l}\text { Lives alone, ex-wife cares for him, } \\
\text { speech problems }\end{array}$ & $\begin{array}{l}\text { High blood pressure, high cholesterol, stroke, } \\
\text { peripheral arterial disease, combined mitral valve } \\
\text { defect }\end{array}$ \\
\hline $\begin{array}{l}80 \text { years, male } \\
\text { (see online supplementary file 10) }\end{array}$ & Retired, no further information & $\begin{array}{l}\text { High blood pressure, high cholesterol, atrial } \\
\text { fibrillation, chronic heart failure, diabetes mellitus } \\
\text { with kidney failure and cerebral microangiopathy, } \\
\text { coronary heart disease, multicausal gait } \\
\text { disturbance, sleep apnoea }\end{array}$ \\
\hline
\end{tabular}

GPs, general practitioners.

\section{Evidence search and analysis}

The guideline search found 27 German (language), up-to-date, evidence-based and consensus-based CPG addressing diagnoses and symptoms that constitute multimorbidity in the case vignettes. The number of guidelines to be considered per case vignette varied between 2 and 22. Between 59 and 320 (average 138) of the guideline recommendations with potential relevance for a respective case vignette were extracted. These data extractions were distilled into a case specific guideline synopsis not exceeding two pages. The guideline synopses were added to the case vignette.

\section{Adding clinical expertise}

Eighteen GPs of the discussion forum were interested in contributing to the project. They received the 10 case vignettes and guideline synopses along with the questionnaires. Completed documents were finally returned from seven participants (three female and four male GPs). The GP panel's answers to the open questions complemented the guideline recommendations by putting an explicit focus on the cases' psychosocial, cultural and familial backgrounds. Upholding the patients' autonomy was considered a particularly primary goal for managing patients with multimorbidity. The GP panel stated that they considered the recommendations from the guideline synopses when answering the three main questions but rated them as only partially helpful.

\section{Developing $\mathrm{N}$-of-one guidelines}

The case vignettes themselves, the case-based guideline synopses and the clinical evaluation by primary care physicians formed the basis for the algorithmic display of primary care processes for each case vignette. In total, 10 algorithms (see online supplementary files 3-11) were created that depict the cognitive and decision-making processes, which GPs and patients work through during a consultation. Figure 2 gives one example.

All 10 case-specific algorithms served as intermediate steps for the development of the generic 'meta-algorithm' (figure 3).

\section{Patient's Perspective}

Nine relevant research projects were identified and analysed in the literature analysis (six qualitative studies and three quantitative studies).$^{30-39}$ Their results were merged with the results of the qualitative interviews as described above. 


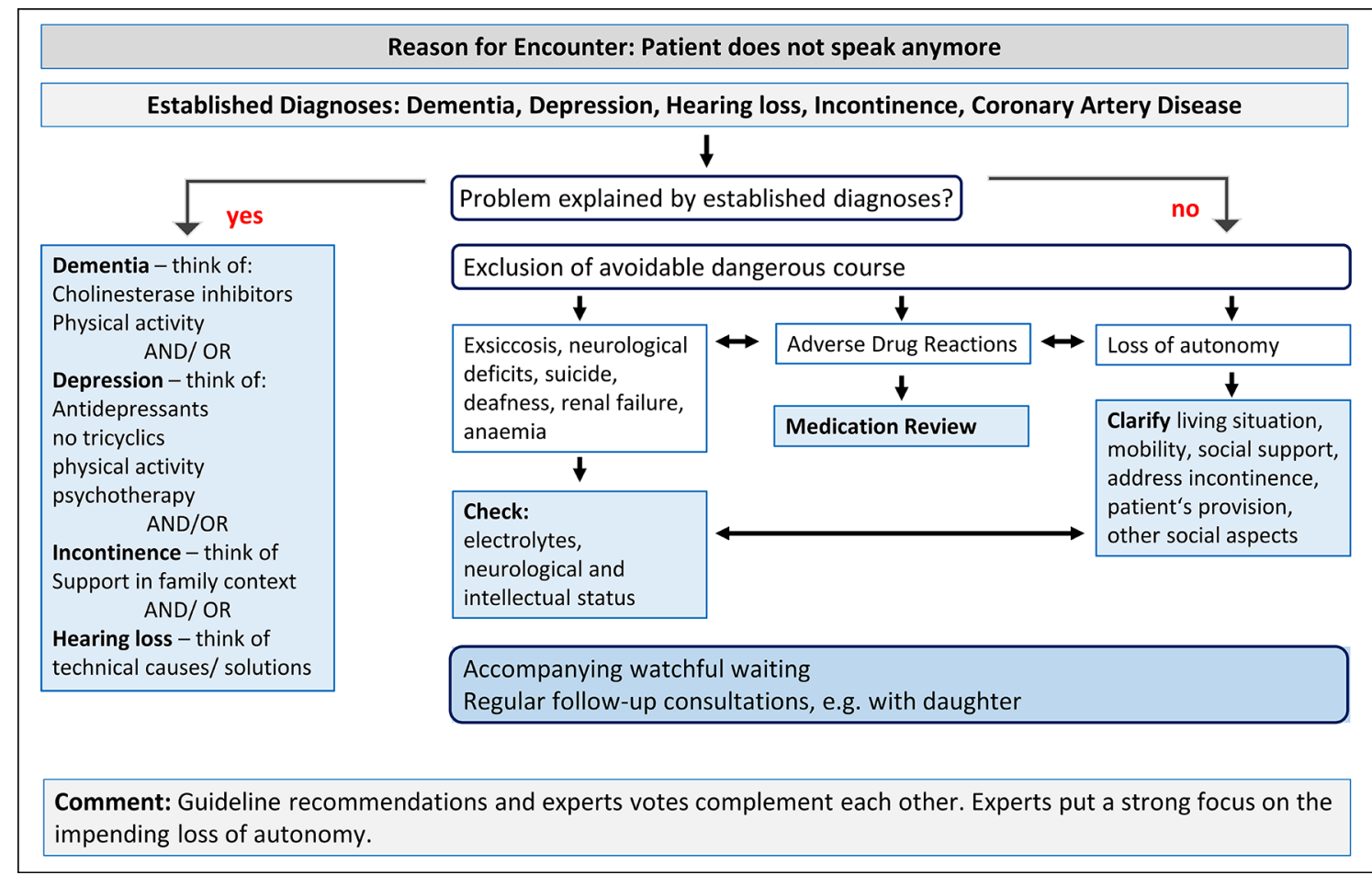

Figure 2 Case-specific algorithm (N-of-one guideline): The 91-year-old patient with multimorbidity presents to his family physician accompanied by his daughter. The reason for encounter is: the patient does not speak anymore. Against the background of established diagnosis, the GP has to decide whether the new symptom is explained by the known diagnoses. If so, progress will be made towards improved disease management. If not, exclusion of an avoidable dangerous course will be prioritised. GP, general practitioner.

Patients' preferences, as expressed in the qualitative studies, were grouped into five main categories: doctor-patient relationship, subjective healthcare needs, communication, organisational framework of healthcare and treatment goals. The categories were not independent of each other. The organisational context of healthcare (such as health insurance, access and availability of providers) forms the basis for all other categories. Communication enables the build-up of a doctor-patient relationship as well as the expression of needs and the formulation of healthcare goals. The results from the quantitative studies as well as the interviews ${ }^{40}$ were fitted into these categories.

Patients with multimorbidity want to be seen and treated as individuals and want to participate in the decision-making process regarding their healthcare. Patients expect their GPs to display honesty and a certain amount of authority, in combination with supplying sufficient information and demonstrating openness for alternative approaches to care. At the same time, respect for patients' psychosocial backgrounds and involvement of the patients' families and friends were highly valued. Formal aspects that contribute to a good doctor-patient relationship were named: sufficient time for the consultation and the embedding of a practice into a healthcare network that facilitates access to specialist care as well as to the non-physician therapeutic professions (eg, physiotherapy). Among the therapeutic goals, patients prioritised the ability to lead an autonomous life. From their point of view, cognitive functioning and mobility are pivotal for autonomy, followed by other functional outcomes. The importance of continuous care was repeatedly mentioned, including means of quickly reacting to health changes or deterioration. Patients with multimorbidity furthermore expressed their preparedness to actively work on achieving care goals together with their physicians (Mundt R, Dissertation Medical Faculty of Hamburg University, in progress).

Preferences and values expressed by patients were considered modifying components during the process of establishing the meta-algorithm.

\section{Synthesis of the meta-algorithm}

Review of the $10 \mathrm{~N}$-of-one guidelines identified a number of common elements: every vignette sets out with a reason for the current encounter. Since GPs typically provide long-term care to their patients and also take on a coordinating role, the reason for the current encounter is viewed against the background of the patient's long-term medical history, the so called 'shared medical history'. The shared medical history consists of the factual information on established diagnoses and symptoms as well as of the patient's psychosocial and familial status. Furthermore, a long-standing doctor-patient relationship contributes to the development and knowledge of the patient's values, life goals and preferences for medical care.

In all N-of-one guidelines, the key question was whether the reason for the current encounter could be explained 


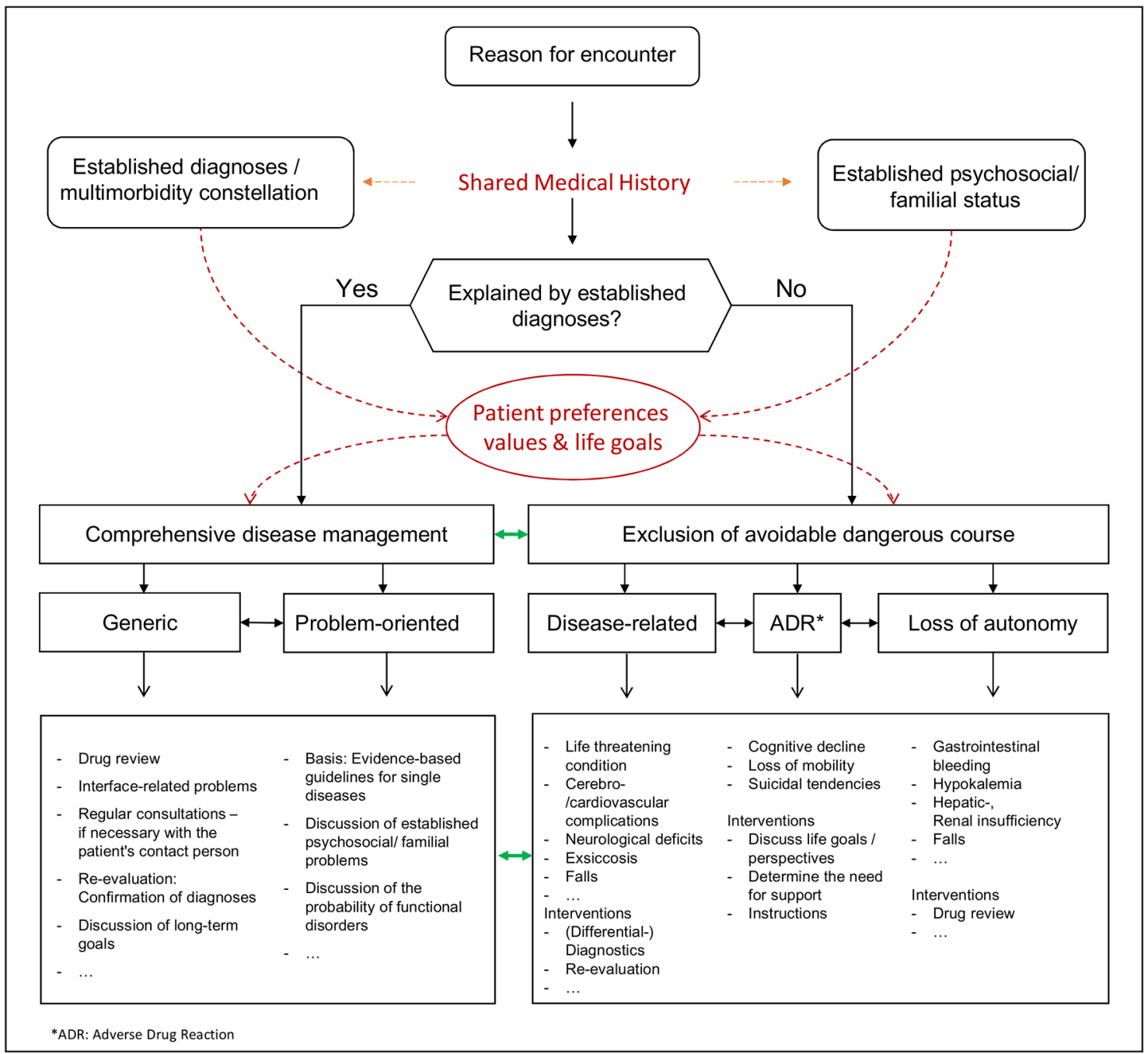

Figure 3 Meta-algorithm to guide the care of patients with multimorbidity in general practice.

by facts known from the shared medical history. 'Yes' results in a comprehensive or problem-oriented disease management with a number of precise but still generic recommendations for action. In case of 'No', investigations on whether an avertable dangerous course of disease can and needs to be avoided are required. Three main and generic foci for these investigations were identified from the N-of-one guidelines: disease-related problems, adverse drug reactions (or interactions) and an impending loss of autonomy. Again, a number of precise but generic recommendations for action were derived from the N-of-one guidelines.

Figure 3 displays the meta-algorithm as a summary of generic considerations derived from 10 case vignettes of patients with multimorbidity in a GP encounter. In the N-of-one guidelines, the key question could not always be answered with a clear-cut 'Yes' or 'No'. Thus, a meta-algorithm must allow to swap from the yes-pathway of actions to the no-pathway and vice versa (green arrows in figure 3).

\section{DISCUSSION}

We outlined the development of a case-based and evidence-based meta-algorithm to guide the management of patients with multimorbidity in general practices.

The algorithm sets out with a patient with multimorbidity presenting with an arbitrary reason for encounter. From this starting point, the cognitive processes that structure the complex consultation situation are displayed. The consideration of patients' preferences, values and life goals stands in the centre of the algorithm and prompts shared decision making, if desired. Priority setting for either disease management or exclusion of an avoidable dangerous course is determined by the answer to one single key question. Both possible pathways are completed by generic recommendations of medical and social aspects to be covered, possible diagnostic, therapeutic and management steps to be taken and information resources to be used. The whole process is embedded in the typical GP setting with a longstanding patient-doctor relationship as the basis for a 'shared medical history'. On the whole, the meta-algorithm encompasses the main criteria for patient-centred 
care. $^{14} 4142$ The meta-algorithm was distilled from 10 evidence-based and consensus-based N-of-one guidelines dealing with the GP management of 10 patients with heterogeneous multimorbidity (case vignettes). The 10 case vignettes were constructed to represent the most frequently encountered disease combinations as well as particularly challenging complex situations presented by practicing GPs. The N-of-one guidelines were developed by the guideline working group, a panel of GPs and methodologists.

As a whole, the meta-algorithm reflects the logic of a GP encounter of a patient with multimorbidity regarding explicit aspects to consider, decision situations and communication needs and priorities. It can be filled with the complex problems of individual patients and hereby offer guidance to the individual practitioner. Contrary to simple, symptom-oriented algorithms, the 'meta-algorithm' illustrates a superordinate process that permanently considers all aspects of a patient. The decision-making processes are primarily guided by the reason for encounter, not by specific diagnoses or combinations of diagnoses. Naturally, avoiding an avertable, dangerous course of disease is a main priority in GP care, provided it is compatible with the individual patient's values and preferences. Especially avoiding the loss of autonomy and maintaining independence (in the sense of the patients' abilities to lead their own lives) has gained new priority as could be gathered from the $\mathrm{N}$-of-one guidelines and the information regarding patients' preferences. This goal may-in individual cases-even supersede solely disease-oriented guideline-based management decisions. Still, disease-specific, evidence-based and guideline-based recommendations play a pivotal role in disease management, if embedded in the holistic care process.

The meta-algorithm in its final form was consented by the multidisciplinary guideline group that is led by the DEGAM.

\section{Strengths and weaknesses of the methodological approach}

To our knowledge, this is the first study attempting a bottom-up approach based on case vignettes of real patients to develop a comprehensive algorithm for managing of patients with multimorbidity. We are aware that 10 patients cannot be representative for all patients with multimorbidity in German general practices. For practical reasons, we restricted our work to 10 patient vignettes that can at least be considered typical patients/ situations. Seven out of 10 case vignettes obtained common disease combinations from two German epidemiological studies. ${ }^{25} 26$ Three further case vignettes representing particularly challenging patients were developed from real cases presented by the participating GPs in the workshop. As another strength, we regard the development of the N-of-one guidelines, because it followed a standard guideline development methodology for evidence-based and consensus-based CPG.

To make sure that the recommendations in the $\mathrm{N}$-of-one guidelines are evidence based, a comprehensive search for German evidence-based and consensus-based guidelines was performed. Further quality assessment was waived since all retrieved guidelines were of accredited high-quality (S3-Standard, according to AWMF). ${ }^{43}$ The prioritisation of recommendations within the development of the $\mathrm{N}$-of-one guidelines was reached by consensus within the guideline working group.

An external GP panel was recruited via an email discussion forum to clinically validate the cases. This small sample of seven GPs is likely a positive selection since participants in this forum have an above-average interest in improving primary care. This disadvantage has to be accounted for in the pilot study, which needs to include a larger and more representative sample of GPs in Germany.

A literature review as well as qualitative interviews were conducted in order to assess the patients' preferences and values regarding the care received through their GPs. The literature search for the review was purposefully kept very specific by using 'multimorbidity' as the main search term because we intended to include research that perceives 'multimorbidity' as a unique entity instead of comorbidities accompanying a specific index disease. The fact that the results from the qualitative interviews of patients with multimorbidity corresponded well with the results from the literature analysis made us confident that we actually captured the main aspects of the patient perspective.

The meta-algorithm is going to form the centrepiece of the multimorbidity guideline of the DEGAM and has been consented in a formal consensus process for this purpose. The algorithm will be embedded in concrete evidence-based and/or consensus-based recommendations concerning communication, management and coordination of care and infrastructural context in the guideline document. The meta-algorithm could, in itself, be useful in structuring primary care encounters outside Germany since it does not refer to a specific infrastructural context.

\section{Comparison with the literature}

Among clinicians but also in the research community there is a consensus that patients with multimorbidity and their multifold healthcare needs pose a major challenge to primary care physicians who are often overwhelmed by the complexity of problems. ${ }^{44-46}$ Furthermore, it is agreed that following the recommendations of every applicable CPG for single disorders is neither feasible nor reasonable, taking into account the resulting treatment burden and numerous possible adverse interaction effects. ${ }^{11} 4748$ Still, the evidence on the effectiveness of interventions to improve the outcomes of patients with multimorbidity is rather limited. A recent Cochrane Review ${ }^{49}$ reported the effectiveness of organisational (12 randomised controlled trials (RCTs)) and patient-oriented (six RCTs) interventions to improve the outcomes of patients with multimorbidity in primary care and community settings. The authors conclude that there is a good amount of uncertainty remaining as concerns the effectiveness of interventions due to the relatively small number of studies 
available to date and their mixed results. An improvement of the evidence base is to be expected though since the authors identified 15 ongoing trials. Interventions like the meta-algorithm, which would be classified as a professional intervention, ${ }^{50}$ were not addressed in the review. Still, one of the conclusions the authors of the Cochrane Review came to was that, in order to achieve sustainability, interventions have to integrate with the existing healthcare system. A requirement that is met by this meta-algorithm.

So far there is still only one CPG dealing explicitly with the management of patients with multimorbidity: the guideline 'Multimorbidity: clinical assessment and management' issued by the NICE. ${ }^{17}$ The guideline was developed via the standard 'top-down' approach to guideline development and is based on extensive literature analyses. The NICE guideline addresses not only primary care providers but all healthcare professionals, including both generalists and specialists. Our meta-algorithm and the NICE guideline do not contradict but complement each other: the meta-algorithm guides clinical reasoning for every GP encounter from a holistic perspective. Medical and psychosocial information from the shared medical history as well as patients' preferences, values and life goals communicated in a long-standing doctorpatient relationship back-up and guide priority setting in every new encounter. The meta-algorithm offers guidance to GPs in steering through complex clinical situations and identifying high priority problems while, at the same time, not losing sight of their complexity. The NICE guideline offers a large number of detailed recommendations while lacking the clinical reasoning structure in primary care. The latter may be due to the fact that the NICE guideline is not confined to primary care but addresses all participants in healthcare as well as patients, their relatives and caregivers.

Muth et al present the 'Ariadne principles' resulting from an expert workshop and two extensive discussion and feedback rounds among GPs and other experts for multimorbidity in primary care from six countries in North America, Europe and Australia. The principles reflect the core elements of an ongoing counselling process for patients with multimorbidity. ${ }^{15}$ The elements of the Ariadne principles are also found in our meta-algorithm: clarifying interactions may be part of accompanying disease management or part of investigating the avoidability of a dangerous disease course.Respecting patient preferences and the mutual agreement on treatment goals are basic principles for any doctor-patient interaction at the various decision points of the algorithm. The main difference between the two concepts is that the meta-algorithm structures one specific consultation. The reason for the encounter determines priorities for the current consultation, while other aspects are posteriorised and maybe postponed to the next encounter. In this way, the meta-algorithm helps to keep a holistic view on the care of patients with multimorbidity and at the same time prevents overloading the current consultation session.
Another intervention, which has some similarities to the proposed meta-algorithm, has been pilot-tested in an exploratory cluster randomised trial (CARE Plus study). The intervention, which is termed a 'whole-system-intervention', was applied in primary care practices in Glasgow and addressed patients with multimorbidity from deprived areas. It consists of longer and structured primary care consultations, the establishment of a care plan and selfhelp support (CARE Approach). The exploratory trial demonstrated positive effects on some endpoints (negative well-being and quality of life) and indicated that the intervention was cost-effective. ${ }^{5152}$

Yet another approach is currently being tested in a pragmatic randomised controlled trial. The three-dimensional (3D) study is testing the effectiveness of a novel approach to GP management of patients with multimorbidity compared with usual care. The intervention is based on a conceptual framework incorporating the patient-centred care model and aims at improving patients' quality of life, reducing the burden of illness and treatment and improving patients' care experiences. GPs received specific training and incentives to foster the implementation of the intervention. The trial is scheduled to end in May 2017 (http://www.isrctn.com/ISRCTN06180958). The intervention of the 3D study has some overlaps with the meta-algorithm, especially in the emphasis on the continuity of care, the coordinated holistic review (instead of disease-focused review) and the focus on patients' priorities and needs, quality of life and function and disease management. 3D study furthermore focuses on detecting depression, which is not explicitly addressed in the meta-algorithm. ${ }^{53}$

To prepare the implementation of the meta-algorithm, it will be embedded into the CPG 'Multimorbidity' of the DEGAM. The guideline itself will be pilot-tested in a sample of GP practices in northern Germany with a primary focus on feasibility and practicability. After necessary modifications are made based on the evaluation results, a quantitative evaluation is planned to investigate the process and patient outcomes. Therefore, the implementation of the meta-algorithm will ideally be complemented by the use of a classification system such as the International Classification of Primary Care. This system can be used to document multiple episodes of care in one patient over time. ${ }^{54}$

\section{CONCLUSION}

The case-based and evidence-based meta-algorithm presented here provides guidance on handling multimorbidity in primary care. It incorporates the principles of patient-centred care. The bottom-up development based on N-of-one guidelines was based on research evidence as well as on GPs' clinical expertise. Applying the meta-algorithm will enable individualised evidence-based care. The next steps will incorporate the implementation and testing of the meta-algorithm in practices as a part of a CPG.

Correction notice This paper has been amended since it was published Online First. Owing to a scripting error, some of the publisher names in the references 
were replaced with 'BMJ Publishing Group'. This only affected the full text version, not the PDF. We have since corrected theseerrors and the correct publishers have been inserted into the references.

Acknowledgements We would like to thank Stefanie Butz, MPH, for conducting the guideline searches and extractions. We would also like to thank Dr Christiane Muth, MPH, and Martin Beyer for constructive discussions within the guideline development process. Also, we would like to thank all participating GPs and patients for their contributions.

Collaborators Guideline group: Heike Hansen, Hendrik van den Bussche and Hans-Hermann Dubben: Institute for Primary Care and Family Medicine, University Medical Center Hamburg-Eppendorf (UKE), Germany. Joachim Szecsenyi: Department of General Practice and Health Service Research, University Heidelberg, Germany. Wolfgang von Renteln-Kruse: Albertinen-Krankenhaus und AlbertinenHaus, Center for Geriatry and Gerontology Hamburg, Germany. Rüdiger Thiesemann: German Society of Gerontology and Geriatrics, Berlin, and University of Witten Herdecke, Chair of Geriatrics. Werner Hofmann: Klinik für Frührehabilitation und Geriatrie Bad Bramstedt, Germany.

Contributors MS was responsible for conceptual design of the case-based guideline development. IS contributed the epidemiological basis for the case vignettes. All authors contributed to the development of the case vignettes. $\mathrm{H}-\mathrm{OW}$ and MS contributed clinical expertise. CM-B and DL were responsible for the guideline synopsis. $\mathrm{H}-\mathrm{OW}, \mathrm{CM}-\mathrm{B}$ and $\mathrm{DL}$ synthesised the $\mathrm{N}$-of-1-guidelines. RM performed literature analysis and interviews for patient preferences. All authors contributed to the compilation of the meta-algorithms. CM-B and DL drafted the manuscript. The final version of the paper has been reviewed and approved by all authors, including the DEGAM guideline group.

Competing interests None declared.

Ethics approval The Multicare cohort study was approved by the Ethics Committee of the Medical Association of Hamburg (approval no. 2881) on 8 November 2013. All participants gave a written informed consent to participate in the study.

Provenance and peer review Not commissioned; externally peer reviewed.

Data sharing statement There are no unpublished data available for data sharing.

Open Access This is an Open Access article distributed in accordance with the Creative Commons Attribution Non Commercial (CC BY-NC 4.0) license, which permits others to distribute, remix, adapt, build upon this work non-commercially, and license their derivative works on different terms, provided the original work is properly cited and the use is non-commercial. See: http://creativecommons.org/ licenses/by-nc/4.0/

(c) Article author(s) (or their employer(s) unless otherwise stated in the text of the article) 2017. All rights reserved. No commercial use is permitted unless otherwise expressly granted.

\section{REFERENCES}

1. Marengoni A, Angleman S, Melis R, et al. Aging with multimorbidity: a systematic review of the literature. Ageing Res Rev 2011;10:430-9.

2. Schram MT, Frijters D, van de Lisdonk EH, et al. Setting and registry characteristics affect the prevalence and nature of multimorbidity in the elderly. J Clin Epidemiol 2008;61:1104-12.

3. Fortin M, Hudon C, Haggerty J, et al. Prevalence estimates of multimorbidity: a comparative study of two sources. BMC Health Serv Res 2010;10:111.

4. van den Bussche $H$, Schäfer I, Wiese $B$, et al. A comparative study demonstrated that prevalence figures on multimorbidity require cautious interpretation when drawn from a single database. J Clin Epidemiol 2013;66:209-17.

5. Uijen AA, van de Lisdonk EH. Multimorbidity in primary care: prevalence and trend over the last 20 years. Eur J Gen Pract 2008;14(Suppl 1):28-32.

6. France EF, Wyke S, Gunn JM, et al. Multimorbidity in primary care: a systematic review of prospective cohort studies. Br J Gen Pract 2012;62:297-307.

7. Fortin M, Lapointe L, Hudon C, et al. Multimorbidity and quality of life in primary care: a systematic review. Health Qual Life Outcomes 2004;2:51.

8. Gijsen R, Hoeymans N, Schellevis FG, et al. Causes and consequences of comorbidity: a review. J Clin Epidemiol 2001;54:661-74.
9. Wallace E, Salisbury C, Guthrie B, et al. Managing patients with multimorbidity in primary care. BMJ 2015;350:h176.

10. Muth C, Glasziou PP. Guideline recommended treatments in complex patients with multimorbidity. BMJ2015;351:h5145.

11. Boyd CM, Darer J, Boult C, et al. Clinical practice guidelines and quality of care for older patients with multiple comorbid diseases: implications for pay for performance. JAMA 2005:294:716-24.

12. Boyd CM, Lucas GM. Patient-centered care for people living with multimorbidity. Curr Opin HIV AIDS 2014;9:419-27.

13. Boyd CM, Reider L, Frey K, et al. The effects of guided care on the perceived quality of health care for multi-morbid older persons: 18-month outcomes from a cluster-randomized controlled trial. J Gen Intern Med 2010;25:235-42.

14. Scholl I, Zill JM, Härter M, et al. An integrative model of patientcenteredness - a systematic review and concept analysis. PLoS One 2014;9:e107828.

15. Muth $\mathrm{C}$, van den Akker M, Blom JW, et al. The Ariadne principles: how to handle multimorbidity in primary care consultations. BMC Med 2014;12:223.

16. Stott NC, Davis $\mathrm{RH}$. The exceptional potential in each primary care consultation. J R Coll Gen Pract 1979;29:201-5.

17. National Institute for Health and Care Excellence. Multimorbidity: clinical assessment and management | guidance and guidelines | NICE [Internet]. 2016 https://www.nice.org.uk/guidance/ng56.

18. Muth $\mathrm{C}$, Beyer M, Popert U. Hausärztliche Leitlinie multimedikation [Internet]. 2014 http://www.degam.de/files/Inhalte/LeitlinienInhalte/Dokumente/S1-Handlungsempfehlung/053-043I_S2e Multimedikation_2014-05.pdf.

19. Blozik E, Dubben $\mathrm{HH}$, Wagner HO, et al. [Comorbidity in medical guidelines: comparison of the current state, epidemiologic models and expert opinion]. Z Evid Fortbild Qual Gesundhwes 2014;108:219-28.

20. Blozik E, van den Bussche $\mathrm{H}$, Gurtner F, et al. Epidemiological strategies for adapting clinical practice guidelines to the needs of multimorbid patients. BMC Health Serv Res 2013;13:352.

21. Muche-Borowski C, Selmann H, Müller W, et al. AWMF-Regelwerk Leitlinien: Ständige Kommission "Leitlinien" der Arbeitsgemeinschaft der Wissenschaftlichen Medizinischen Fachgesellschaften (AWMF). Arbeitsgemeinschaft der Wissenschaftlichen Medizinischen Fachgesellschaften AWMF. 2014 http://www.awmf.org/leitlinien/ awmf-regelwerk.html.

22. Graham R. Clinical practice guidelines we can trust. Washington, DC: National Academies Press, 2011.

23. Muth $\mathrm{C}$, Kirchner $\mathrm{H}$, van den Akker $\mathrm{M}$, et al. Current guidelines poorly address multimorbidity: pilot of the interaction matrix method. J Clin Epidemiol 2014;67:1242-50.

24. Kravitz R, Duan N. Design and Implementation of $\mathrm{N}$-of-1 Trials: A User's Guide. Rockville (MD: AHRQ, 2014. Report No.: 13(14-EHC122-EF). www.effectivehealthcare.ahrq.gov/N-1-Trials. cfm.

25. Schäfer I, von Leitner EC, Schön G, et al. Multimorbidity patterns in the elderly: a new approach of disease clustering identifies complex interrelations between chronic conditions. PLoS One 2010;5:e15941.

26. van den Bussche $H$, Koller D, Kolonko T, et al. Which chronic diseases and disease combinations are specific to multimorbidity in the elderly? results of a claims data based cross-sectional study in Germany. BMC Public Health 2011;11:101.

27. Schäfer I, Hansen $\mathrm{H}$, von dem Knesebeck $\mathrm{O}$, et al. Welche chronischen krankheiten und morbiditätsmuster charakterisieren die ältere multimorbide Population in der Hausarztpraxis? Z Geronto Geriatr 2011:2:73-98.

28. Giacomini MK, Cook DJ. Users' guides to the medical literature: XXIII. Qualitative research in health care A. Are the results of the study valid? Evidence-Based Medicine Working Group. JAMA 2000;284:357-62.

29. Harbour RT. Scottish Intercollegiate Guidelines Network. SIGN 50: a guideline developer's handbook [Internet. Ediburgh, Scotland: Scottish Intercollegiate Guidelines Network, 2008. http://www.sign. ac.uk/pdf/sign50.pdf.

30. Noël PH, Parchman ML, Williams JW, et al. The challenges of multimorbidity from the patient perspective. J Gen Intern Med 2007;22(Suppl 3):419-24.

31. Fung $\mathrm{CH}$, Setodji CM, Kung FY, et al. The relationship between multimorbidity and patients' ratings of communication. J Gen Intern Med 2008;23:788-93.

32. Kiliari N, Theodosopoulou E, Papanastasiou E. Multimorbidity and unmet citizens' needs and expectations urge for reforms in the health system of Cyprus: a questionnaire survey. JRSM Open 2014;5:2042533313515860. 
33. Jerant AF, von Friederichs-Fitzwater MM, Moore M. Patients' perceived barriers to active self-management of chronic conditions. Patient Educ Couns 2005;57:300-7.

34. Noël PH, Frueh BC, Larme AC, et al. Collaborative care needs and preferences of primary care patients with multimorbidity. Health Expect 2005;8:54-63.

35. Bayliss EA, Edwards AE, Steiner JF, et al. Processes of care desired by elderly patients with multimorbidities. Fam Pract 2008;25:287-93.

36. Fried TR, McGraw S, Agostini JV, et al. Views of older persons with multiple morbidities on competing outcomes and clinical decisionmaking. J Am Geriatr Soc 2008;56:1839-44.

37. Ekdahl AW, Andersson L, Friedrichsen M. "They do what they think is the best for me." Frail elderly patients' preferences for participation in their care during hospitalization. Patient Educ Couns 2010;80:233-40.

38. Robben S, van Kempen J, Heinen M, et al. Preferences for receiving information among frail older adults and their informal caregivers: a qualitative study. Fam Pract 2012;29:742-7.

39. van Kempen JA, Robben SH, Zuidema SU, et al. Home visits for frail older people: a qualitative study on the needs and preferences of frail older people and their informal caregivers. $\mathrm{Br} J$ Gen Pract 2012;62:554-60.

40. Mundt R, Muche-Borowski C, Pohontsch N, et al, 2015. Assessing preferences of patients with multimorbidity in primary care: $\mathrm{A}$ qualitative study. Poster Presentation at the G-I-N Conference. Amsterdam. https://www.eventure-online.com/eventure/public/publicSession. form?id $=250890$ \& test $=250890$

41. Stewart M. Towards a global definition of patient centred care. BMJ2001;322:444-5.

42. American Geriatrics Society Expert Panel on the Care of Older Adults with Multimorbidity. Patient-centered care for older adults with multiple chronic conditions: a stepwise approach from the American Geriatrics Society: American Geriatrics Society Expert Panel on the Care of Older Adults with Multimorbidity. J Am Geriatr Soc 2012;60:1957-68.

43. Nothacker M, Muche-Borowski C, Kopp I. Guidelines in the Register of the Association of Scientific Medical Societies in Germany - A Quality Improvement Campaign. Geburtshilfe Frauenheilkd 2014;74:260-6.
44. Smith SM, Soubhi $\mathrm{H}$, Fortin $\mathrm{M}$, et al. Managing patients with multimorbidity: systematic review of interventions in primary care and community settings. BMJ 2012;345:e5205.

45. O'Brien R, Wyke S, Guthrie B, et al. An 'endless struggle': a qualitative study of general practitioners' and practice nurses' experiences of managing multimorbidity in socio-economically deprived areas of Scotland. Chronic IIIn 2011;7:45-59.

46. Sinnott C, Mc Hugh S, Browne J, et al. GPs' perspectives on the management of patients with multimorbidity: systematic review and synthesis of qualitative research. BMJ Open2013;3:e003610.

47. Greenhalgh T, Howick J, Maskrey N; Evidence Based Medicine Renaissance Group. Evidence based medicine: a movement in crisis? BMJ 2014;348:g3725.

48. Buffel du Vaure C, Ravaud P, Baron G, et al. Potential workload in applying clinical practice guidelines for patients with chronic conditions and multimorbidity: a systematic analysis. BMJ Open 2016;6:e010119.

49. Smith SM, Wallace E, O'Dowd T, et al. Interventions for improving outcomes in patients with multimorbidity in primary care and community settings. Cochrane Database Syst Rev 2016;3:CD006560.

50. Smith S, Bayliss E, Mercer S, et al. How to design and evaluate interventions to improve outcomes for patients with multimorbidity. $J$ Comorb 2013;3:10-17

51. Mercer SW, Fitzpatrick B, Guthrie B, et al. The CARE Plus study - a whole-system intervention to improve quality of life of primary care patients with multimorbidity in areas of high socioeconomic deprivation: exploratory cluster randomised controlled trial and costutility analysis. BMC Med 2016:14:88

52. Bikker AP, Mercer SW, Cotton P, et al. Connecting, assessing, responding and empowering (CARE): a universal approach to person-centred, empathic healthcare encounters. Educ Prim Care 2012;23:454-7.

53. Man MS, Chaplin K, Mann C, et al. Improving the management of multimorbidity in general practice: protocol of a cluster randomised controlled trial (The 3D study). BMJ Open 2016;6:e011261.

54. Laux G, Kuehlein T, Rosemann T, et al. Co- and multimorbidity patterns in primary care based on episodes of care: results from the german CONTENT project. BMC Health Serv Res 2008;8:14. 